\title{
A complex double translocation involving four chromosomes and five breakpoints in a child with mild mental retardation
}

\author{
D A COUZIN, J L WATT, AND I A A UCHTERLONIE \\ Departments of Genetics and Child Health, University of Aberdeen, Medical School Buildings, Foresterhill, \\ Aberdeen $A B 92 Z D$.
}

SUMMARY A 6-year-old boy with speech delay and mild mental retardation (IQ 82) was found to have a complex double translocation involving four chromosomes and a total of five breakpoints, two being on the same arm. This resulted in the karyotype $46, \mathrm{XY}, \mathrm{t}(2 ; 4 ; 7)(7 ; 8)-$ (q14;q31;q11q22;q13). As far as the authors are aware this is the first time that such a complex double translocation has been reported. Both parents had normal karyotypes.

There have been very few published cases of complex chromosomal rearrangements involving three chromosomes. ${ }^{1}$ Complex structural rearrangements involving more than three chromosomes are extremely rare. Martinetti and $\mathrm{Noel}^{2}$ reported a slightly dysmorphic newborn child with structural rearrangements involving four chromosomes and consisting of a double (four break) reciprocal translocation of chromosomes 1,5 , and 9 in addition to a pericentric inversion of chromosome 8 . A severely retarded child has also been reported ${ }^{3}$ with a karyotype exhibiting a complex rearrangement between chromosomes $\mathrm{X}$ and 1 with four breakpoints in addition to a reciprocal translocation between chromosomes 7 and 13. Both of these reports involved two separate and distinct rearrangements.

We report here what we believe to be the first case of what might be called a complex double translocation involving four chromosomes and five breakpoints.

\section{Case report}

This boy presented with speech delay at the age of 6 years to the paediatric clinic. Although reasonably numerate he had reading difficulties at school and was receiving remedial help. His IQ on the Stanford Binet scale was 82 (below normal). Both parents were professional and aged 35 .

The mother reported a normal pregnancy and birth with no problems either in the newborn period

Received for publication 1 February 1983. Accepted for publication 10 March 1983. or during pregnancy. There was no known exposure to $x$-rays, drugs, or infection during pregnancy.

On physical examination the child had an odd facies with prominent, cave-like ears which were marginally low set. He had bilateral inner epicanthic folds but the bridge of his nose was rather narrow. The dermatoglyphs were normal except for a curious quarter moon fold just under the metacarpal-phalangeal joint area on the right hand side. There was bilateral clinodactyly. The third and fourth toes on both sides exhibited syndactyly to a degree somewhat greater than normal. He also had a metatarsus varus deformity of the left foot.

\section{CYTOGENETICS}

Conventional lymphocyte cultures were made from the peripheral blood of this boy and both parents. The chromosomes were $\mathrm{G}$ banded ${ }^{4}$ and $\mathrm{C}$ banded. ${ }^{5}$ Both parents were shown to have normal karyotypes as, incidentally, had a previous stillbirth. The child's karyotype on the other hand was grossly abnormal (fig 1). There was only one normal homologue of chromosomes 2, 4, 7, and 8, together with four derived chromosomes. The breakpoints appeared to be at bands $2 \mathrm{q} 14,4 \mathrm{q} 13$, and $8 \mathrm{q} 13$, with two other breakpoints on the long arm of chromosome 7 at bands q11 and q22.

This structural rearrangement can most conveniently be described as being the result of two separate events (fig 2). There is a complex translocation involving chromosomes 2,4 , and 7 with the distal part of the long arm of 2 (q14 $\rightarrow$ qter) being attached to the proximal part of the long arm of 4 , the distal long arm of 4 (q31 $\rightarrow$ qter) being attached to the proximal long arm of 7 , and the translocation being completed by the distal long arm of 7 (q11 $\rightarrow$ qter) being attached to the proximal long arm of 2 . In addition to this event there has apparently occurred what might be considered a simple reciprocal translocation between the distal part of the long arm of 7 (q22 $\rightarrow$ qter) which is translocated onto chromosome 2 and the distal part of the long arm of 8 (q13 $\rightarrow$ qter). This gives the appearance of an interstitial insertion of $7 \mathrm{q} 11 \rightarrow 7 \mathrm{q} 22$ in the $\operatorname{der}(2)$ chromosome at band $2 \mathrm{q} 14$ proximal to the translocated arm of 8 (q13 $\rightarrow$ qter). 

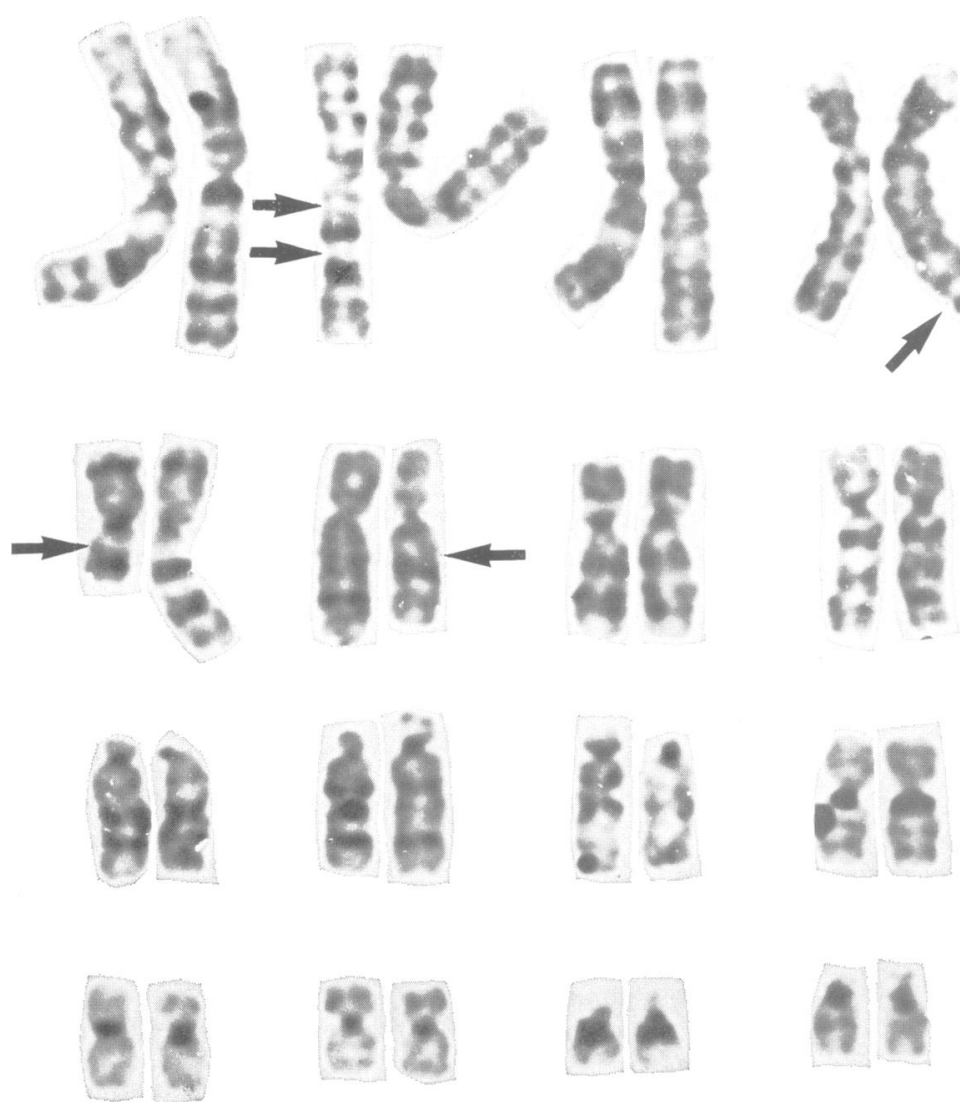

FIG 1 Karyotype of proband $46, X Y, t(2 ; 4 ; 7)(7 ; 8)$ ( $G$ banding).

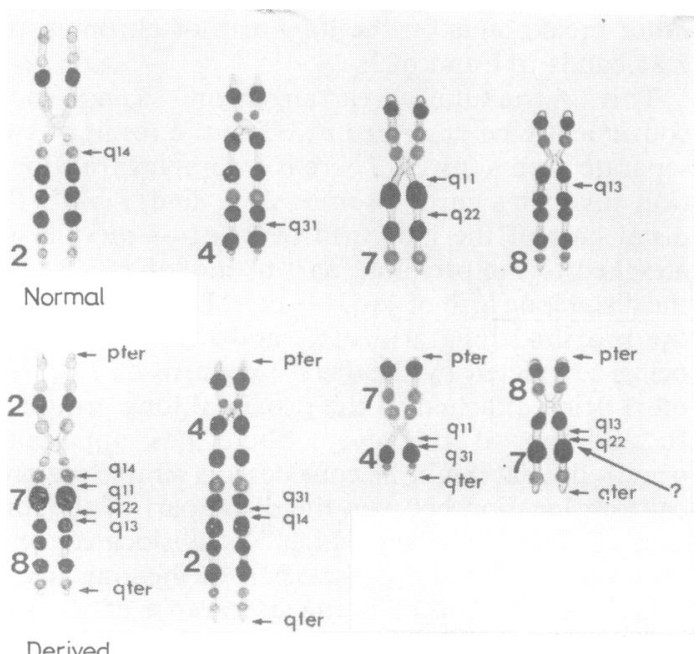

Derived

FIG 2 Diagram illustrating the five breakpoints involved in the complex double translocation.
The detailed designation of this karyotype, following the ISCN (1978) convention, ${ }^{6}$ is then:0 $46, \mathrm{XY}, \mathrm{t}(2 ; 4 ; 7)(7 ; 8)(2 \mathrm{pter} \rightarrow 2 \mathrm{q} 14:: 7 \mathrm{q} 11 \rightarrow 7 \mathrm{q} 22::$ 음 $8 \mathrm{q} 13 \rightarrow 8$ qter $; 4$ pter $\rightarrow 4$ q31 $:: 2$ q14 $\rightarrow 2$ qter $; 7$ qter $\rightarrow \underset{\text {. }}{ }$. $7 \mathrm{q} 11:: 4 \mathrm{q} 31 \rightarrow 4 \mathrm{qter} ; 8 \mathrm{pter} \rightarrow 8 \mathrm{q} 13:: 7 \mathrm{q} 22 \rightarrow 7 \mathrm{qter}$ ).

The nomenclature for the derived karyotype is a hybrid between the ISCN convention for complex 음 translocations (involving three chromosomes and $>$ three breaks) and for double translocations (involv- 음. ing three chromosomes and four breaks). Such a hybrid nomenclature seems to be necessary to $\%$ represent translocations involving $n$ chromosomes $N$ with $\mathrm{n}+1$ breaks where $\mathrm{n}>3$.

When the karyotype was rearranged to reconstruct $\mathcal{O}$ a normal karyotype a residual banding abnormality appeared to remain. This involved the putative $\overparen{\varnothing}$ simple translocation between the rearranged $7 \mathrm{q}$ arm $\stackrel{?}{?}$ on the der(2) chromosome and the $8 \mathrm{q}$ arm (fig 3). This may be the result of a slight imbalance owing to a small partial trisomy of $7 q$ and a concomitant small $\cong$ partial monosomy of $8 \mathrm{q}$. 

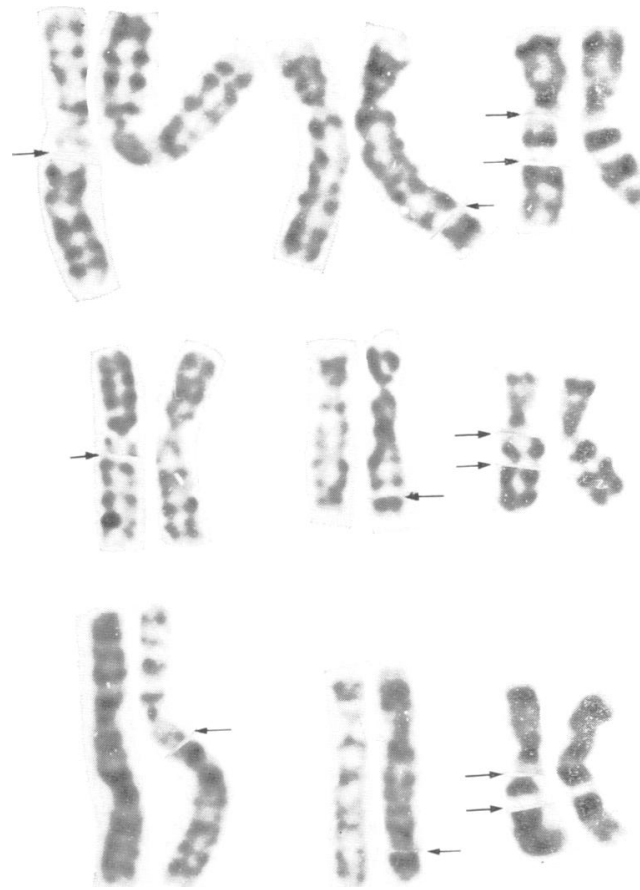

\section{Discussion}

Complex translocations can only be considered as single events in which three lesions occur, one on each of three chromosomes, at the same locality in the cell at more or less the same time. On the other hand four break translocations (or double translocations), where two lesions occur on a single chromosome and one lesion on each of two other chromosomes, could either be associated with a single event causing the four lesions or with two events separated in time and locality, each being associated with two lesions. In the latter case the common involvement of one of the chromosomes must be considered to be fortuitous.

The case reported here exhibited five lesions leading to exchanges. It is quite possible, if not likely, that three of these lesions were associated with one event leading to a complex translocation, while the other two were associated with a second event leading to a simple reciprocal translocation. The final result is a double translocation involving a complex and a simple reciprocal translocation, that is, a complex double translocation. Such mechanistic likelihoods are not apparent from the ISCN nomenclature which merely gives the briefest unambiguous representation of the final state of the derived chromosomes. We suggest that perhaps the ISCN is not adequate in this respect and needs modification to accommodate a greater degree of complexity.

The structural rearrangements in this case must have occurred in a parental gamete or at a very early stage of embryogenesis, since no normal karyotypes were found in 100 cells.

Perhaps the most remarkable fact about this case is the lack of any really notable clinical features. Those features which were observed did not appear to be associated with any reported cases involving abnormalities of the chromosomes being considered here. This may not be entirely surprising if the rearrangements are considered to be completely balanced.

In such a balanced situation, the slight phenotypic abnormalities observed could simply be the result of some position effect or genetic change at the sites of breakage $^{7}$ or may be unrelated to the chromosome rearrangements, yet having a common cause. ${ }^{8}$ Radiation seems a possible candidate but there was no known exposure of germ cells or early zygote.

It seems possible however that the rearrangements were not entirely balanced even at the microscopical level. The suggested trisomy of $7 \mathrm{q}$ and the concomitant monosomy of $8 \mathrm{q}$ are tiny and may explain the small phenotypic effect.

The most likely explanation, then, is that this was a spontaneous occurrence not associated with any particular aetiology and that the possibly unbalanced 


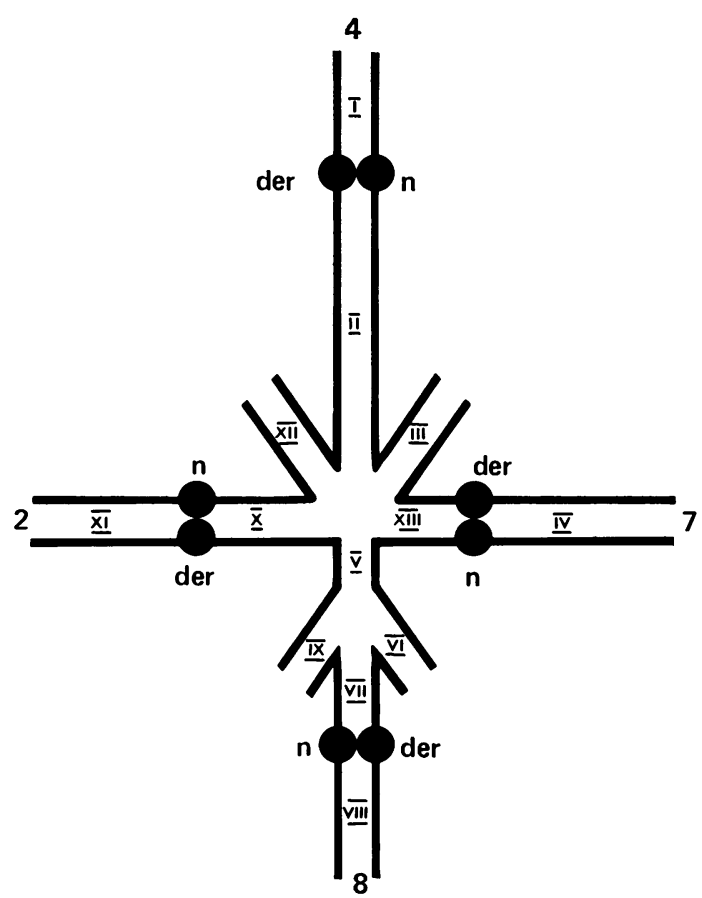

FIG 4 Possible pachytene configuration.

karyotype and slightly abnormal phenotype are in some way related.

Meiosis, if it occurs, is likely to be an extremely complicated affair. Assuming that spermatogenesis is possible, we can theoretically predict the pachytene configuration seen in fig 4 or possibly a chain equivalent, since pairing may be incomplete at the shortest regions of homology. Even without the added complications of cross-over events, but assuming sufficient terminal chiasmata to hold the configuration together, alternate and adjacent disjunctions are expected to give rise to 70 different types of sperm, only two of which will be balanced. The possibilities of $7: 1,6: 2$, and $5: 3$ disjunction must also be considered, since $3: 1$ disjunction is often found in ordinary translocations. ${ }^{9}$ However, strictly speaking, one cannot make such simple assumptions. A more realistic estimate of the make-up of the gamete population would involve consideration of the 13 separate segments which can potentially pair and form zero, one, or two chiasmata. It may be fossible to estimate the relative frequencies of such possibilities based on available chiasmata maps, since we have accurately pinpointed the breakpoints. For the five interstitial segments (II, V, VII, VIII, and X) one would have to consider further the possibilities of two, three, and four strand double cross-overs. These data should allow the frequency of univalents, bivalents, trivalents, etc, to be calculated, although this would seem to be a purely academic exercise.

Many of the abnormal gametes will be inviable because of the extent of the resultant duplication and deficiency, but the odds against producing a normal viable sperm would be extremely low. The overall number and variety of problems appears to make infertility or reproductive failure a virtual certainty.

Buchanan et $a l^{10}$ listed 19 cases of complex chromosome rearrangements and observed that chromosomes $3,4,5,9,11,13$, and 14 were most frequently observed. Only one chromosome of the four involved in this case (No 4) was in the "high frequency' category. This further illustrates the unique nature of the case and highlights the need for modification of ISCN nomenclature. Perhaps the suggestion of a 'complex double translocation' can act as a precedent for future cases of similar complexity.

The authors are grateful to Dr D P Fox for reviewing the manuscript.

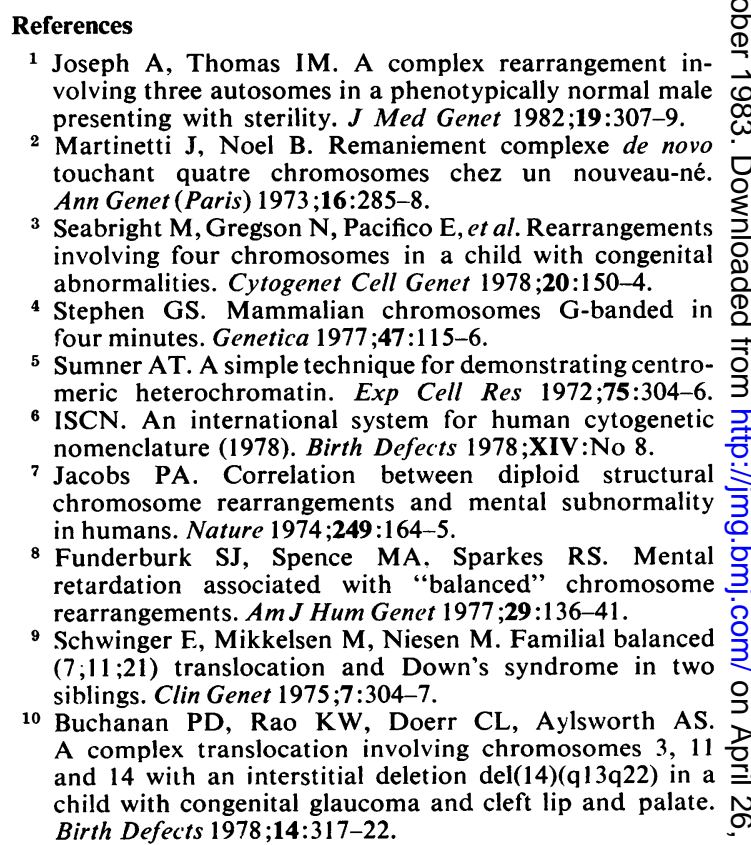

Correspondence and requests for reprints to Dr D Couzin, Department of Genetics, University of Aberdeen, Medical School Buildings, Foresterhill, ? Aberdeen AB9 2ZD.

\section{Note added in proof}

It is conceivable that the residual banding abnormality on chromosome 8 (fig 3 ) is a small paracentric $\frac{\vec{D}}{\mathbb{D}}$ inversion. This possibility would necessitate an additional breakpoint at $8 \mathrm{q} 23$. 